\title{
The Kinematically Measured Pattern Speeds of NGC 2523 and NGC 4245
}

\author{
P. Treuthardt and R. Buta
}

Department of Physics \& Astronomy, University of Alabama, Tuscaloosa, AL 35487, USA

\begin{abstract}
We have applied the Tremaine-Weinberg method to the SB(r)b galaxy NGC 2523 and the SB(r)0/a galaxy NGC 4245 using the Calcium Triplet in order to determine their bar pattern speeds. Assuming an inclination of $53^{\circ}$ and a distance of $49.5 \mathrm{Mpc}$, the pattern speed of NGC 2523 is $26.2 \pm 6.1 \mathrm{~km} \mathrm{~s}^{-1} \mathrm{kpc}^{-1}$. The pattern speed of NGC 4245 is $75.3 \pm 31.2 \mathrm{~km} \mathrm{~s}^{-1}$ $\mathrm{kpc}^{-1}$, assuming an inclination of $35.4^{\circ}$ and a distance of $12.7 \mathrm{Mpc}$. The ratio of the corotation radius to the bar radius of NGC 2523 and NGC 4245 is $1.3 \pm 0.3$ and $1.1 \pm 0.5$, respectively.
\end{abstract}

Keywords. galaxies: bars, galaxies: kinematics and dynamics

Tremaine \& Weinberg (1984, ApJ, 282, L5) derived a kinematic method for estimating the pattern speed, $\Omega_{P}$, of a galaxy from the luminosity weighted mean line-of-sight velocities, $\langle\mathrm{V}\rangle$, and luminosity weighted mean positions, $\langle\mathrm{X}\rangle$, of a tracer that obeys the continuity equation. If these quantities are measured along lines parallel to the major axis of a galaxy, they can be related as $\Omega_{P} \sin i=\langle\mathrm{V}\rangle /\langle\mathrm{X}\rangle$, where $i$ is the inclination of the galaxy. A maximum disk, or fast bar, can be determined from the distance independent ratio, $\mathcal{R}$, of the corotation radius to the bar semimajor axis length. When $\mathcal{R}<1.0$, self-consistent bars cannot exist (Contopoulos 1980, A\&A, 81, 198). A disk is maximal and a bar is considered fast when $1.0 \leqslant \mathcal{R} \leqslant 1.4$ (Debattista \& Sellwood 2000, ApJ, 543, 704). A submaximal disk, or slow bar, occurs when $\mathcal{R}>1.4$. Long-slit spectral observations of the Ca II IR triplet of NGC 2523 and NGC 4245 were taken on January 18 and 19, 2006 using the RC Spectrograph on the KPNO Mayall 4 meter telescope. Results of these observations are shown in Figure 1. This work was supported by NSF Grant AST 050-7140 to the University of Alabama.
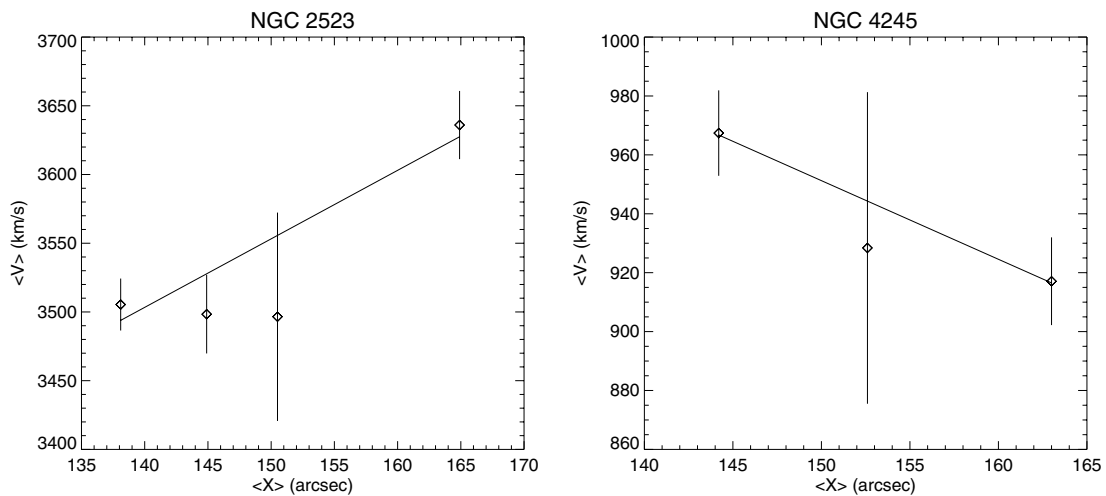

Figure 1. Plots of $\langle\mathrm{V}\rangle$ vs. $\langle\mathrm{X}\rangle$ for NGC 2523 (left) and NGC 4245 (right). The slope and corresponding $1 \sigma$ error that best fits the data is $\Omega_{P} \sin i=5.0 \pm 1.2 \mathrm{~km} \mathrm{~s}^{-1} \operatorname{arcsec}^{-1}$ and $2.7 \pm 1.1 \mathrm{~km} \mathrm{~s}^{-1} \operatorname{arcsec}^{-1}$ for NGC 2523 and NGC 4245, respectively. 